\title{
Terahertz Emission of Gallium Arsenide on Textured p-type Silicon (100) Substrates Grown via Molecular Beam Epitaxy
}

\author{
Karl Cedric P. Gonzales ${ }^{1}$, Karim M. Omambac ${ }^{1}$, Elizabeth Ann P. Prieto ${ }^{1}$, Jessica Pauline C. Afalla ${ }^{1}$, Maria Herminia \\ M. Balgos ${ }^{1}$, Rafael B. Jaculbia ${ }^{1}$, Armando S. Somintac ${ }^{1}$, Arnel A. Salvador ${ }^{1}$, Elmer S. Estacio ${ }^{1}$
}

1National Institute of Physics, University of the Philippines, Diliman, Quezon City, Philippines 1101; cgonzales@nip.upd.edu.ph

\section{A R T I C LE I N F O}

Article history:

Received : October 2018

Accepted : November 2018

Available online : November 2018

\section{Keywords:}

GaAs on Si

Molecular Beam Epitaxy

Terahertz

Surface Texturing

\begin{abstract}
A B S T R A C T
This study presents the terahertz (THz) emission of molecular beam epitaxy (MBE)-grown Gallium Arsenide (GaAs) on surface textured p-type Silicon (pSi) (100) substrates. Surface texturing was achieved by anisotropic wet chemical etching using 5\% wt Potassium Hydroxide (KOH): Isopropyl alcohol (IPA) (50:1) solution for 15, 30, 45, and 60 minutes. Reflectivity measurements for the textured $\mathrm{p}$-Si(100) substrates indicated that the overall texturing increases with longer etching times. Raman spectroscopy of the post-growth samples confirmed GaAs growth. The $\mathrm{THz}$ emission intensities were the same order of magnitude. The GaAs grown on $\mathrm{p}-\mathrm{Si}(100)$ textured for 60 minutes exhibited the most intense THz emission attributed to the increased absorption from a larger surface-to-volume ratio due to surface texturing. All GaAs on textured p-Si(100) samples had frequency bandwidth of $\sim 2.5 \mathrm{THz}$.
\end{abstract}

\section{Introduction}

Since the development of $\mathrm{THz}$ time-domain spectroscopy (THz-TDS), THz technology has been growing rapidly in the past 30 years [1-3]. Recent breakthroughs in this emerging field include $\mathrm{THz}$ imaging, high-power $\mathrm{THz}$ generation via non-linear effects, and compact THz-TDS systems leading to interdisciplinary applications. The main component of these systems are the $\mathrm{THz}$ sources. GaAs-based semiconductors have established their application as $\mathrm{THz}$ sources. Low-temperature GaAs (LTG-GaAs) grown on semi-insulating GaAs (SI-GaAs) have been widely used as effective $\mathrm{THz}$ emitters as photoconductive antennas [4]. LTG-GaAs on SI-GaAs is typically grown at substrate temperatures $\left(T_{s}\right)$ of $200^{\circ} \mathrm{C}-460^{\circ} \mathrm{C}$ resulting to high concentration of defects due to excess arsenic (As) during growth [5]. The intentional incorporation of defects leads to changes in the carrier dynamics within the epitaxial layer dictating the $\mathrm{THz}$ emission efficiency.

GaAs on Silicon (GaAs on $\mathrm{Si}$ ) substrates have recently been gaining a lot of interest as $\mathrm{THz}$ sources due to cost-efficiency of Si substrates as well as its transparency to THz region as compared to SI-GaAs substrates [6]. GaAs on Si integration may lead to relatively low-cost $\mathrm{THz}$ emitters with promising characteristics. The growth of high-quality GaAs on Si often results to high dislocation densities due to the $\sim 4.1 \%$ lattice mismatch between GaAs and Si [7]. Various methods have been reported to reduce the dislocation densities of the GaAs epitaxial layer such as utilizing a GeSi intermediate buffer [8-9], two-step growth $[7,10]$, and thermal cycle annealing [11-12].

In this work, the $\mathrm{THz}$ response from GaAs grown on $\mathrm{Si}$ with varying induced defect concentration is investigated by THz-TDS in reflection excitation geometry. Intentional introduction of defects is implemented by surface texturing of the p-Si(100) substrate prior to the growth of the GaAs epitaxial layers via molecular beam epitaxy (MBE).

\section{Experimental Details}

The surface texturing of the $\mathrm{p}-\mathrm{Si}(100)$ substrates was achieved by performing an alkaline wet chemical etching using $5 \mathrm{wt} \%$ Potassium hydroxide $(\mathrm{KOH})$ pellets diluted in deionized water with isopropyl alcohol (IPA) as wetting agent. The p-Si(100) substrates were submerged in a KOH:IPA (50:1) solution heated at $70^{\circ} \mathrm{C}$ for $15,30,45$ and 60 minutes resulting to varying nanopyramid sizes and densities. Each substrate was rinsed with deionized water and N2 dried.

The textured $\mathrm{p}-\mathrm{Si}(100)$ substrates at varying etching times were mounted onto a single 3" molybdenum block and were loaded into a RIBER 23P MBE system. Oxide removal of the substrates was conducted at $T_{s}=600^{\circ} \mathrm{C}$. A $0.5 \mu \mathrm{m}$ GaAs epitaxial layer was then grown at $T_{s}=590^{\circ} \mathrm{C}$. The growth of the GaAs epitaxial layer was terminated with a 0.02 
$\mu \mathrm{m} n$-doped GaAs cap. The summary of the 4 samples is tabulated in Table 1.

Table 1: Summary of the GaAs on textured p-Si(100) samples with varying substrate etching times

\begin{tabular}{cc}
\hline SAMPLE & $\mathrm{p}$-Si(100) etching time \\
\hline A & 15 mins \\
B & 30 mins \\
C & 45 mins \\
D & 60 mins \\
\hline
\end{tabular}

The surface morphology of the textured p-Si(100) substrates was observed by scanning electron microscopy (SEM) micrographs. The overall surface roughness of the substrates was characterized by reflectivity measurements. The growth of GaAs was confirmed by Raman spectroscopy. The THz emission of the samples were measured by THz-TDS in reflection excitation geometry.

\section{Results and Discussions}

The SEM micrographs of the surface morphology of substrates prior to growth confirmed the surface texturing of the p-Si(100) substrates with wet chemical etching as shown in Fig. 1. Varying sizes and densities of pyramidal structures were observed with varying etching times [13]. A general increase in pyramid sizes and dimensions with increasing etching time was observed. The reflectivity measurements of the samples are shown in Fig. 1. A general decrease in the reflectance with increasing etching times was observed indicating that sample D had the highest absorption due to surface texturing [13]. This decrease in specular reflectance is attributed to the multiple internal reflections as the light trapping mechanism on the rough sample surface.
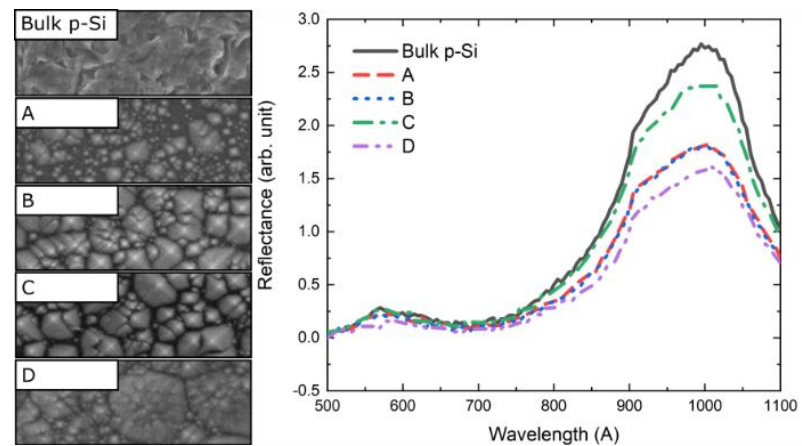

Fig. 1: Reflectivity measurements of the textured p-Si(100) substrates compared to the untextured substrate (right) with their corresponding top view SEM micrographs (left).

The Raman spectra of the GaAs on textured p$\mathrm{Si}(100)$ samples compared with the bulk p-Si(100) subsrate with their corresponding top view SEM micrographs are illustrated in Fig. 2. The bulk p$\mathrm{Si}(100)$ substrate exhibited one peak at $520.41 \mathrm{~cm}-1$, the fundamental Si Raman peak [14]. On the other hand, all the GaAs on textured p-Si(100) samples had two more Raman peaks attributed to GaAs transverse optical (TO) and longitudinal optical (LO) phonon modes indicating the growth of GaAs layers [15].
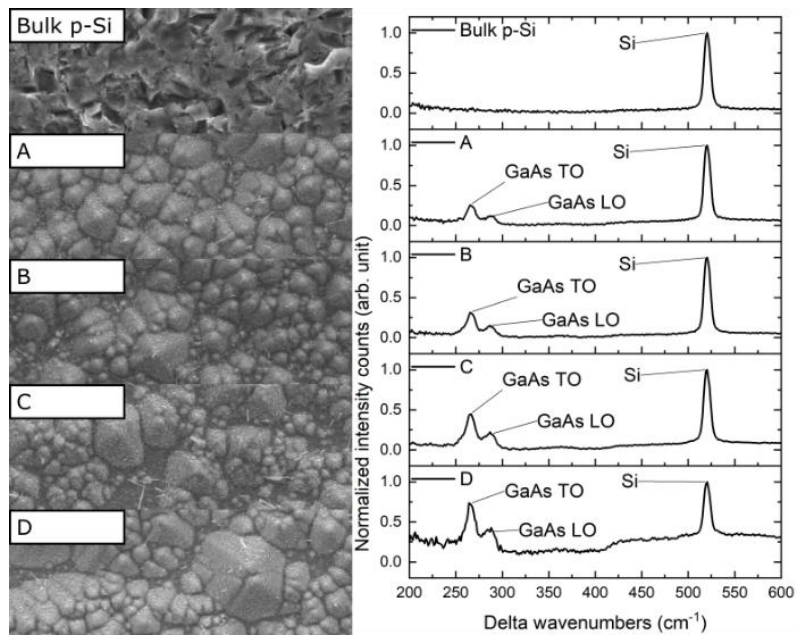

Fig. 2: Raman spectra of GaAs on textured p-Si(100) compared to the untextured substrate (right) with the corresponding top view SEM micrographs (left)

The $\mathrm{THz}$ emission intensities with the corresponding power spectra of all the samples are shown in Fig. 3. The peak-to-peak intensities are listed in Table 2. There was no observed $\mathrm{THz}$ emission from the bulk p-Si(100) substrate indicating that the $\mathrm{THz}$ emission from all the samples was solely from the grown GaAs layers. There was no observed significant difference in $\mathrm{THz}$ emission intensity with increasing substrate texturing time. Furthermore, all GaAs on textured p-Si(100) had frequency bandwidths of $\sim 2.5 \mathrm{THz}$.
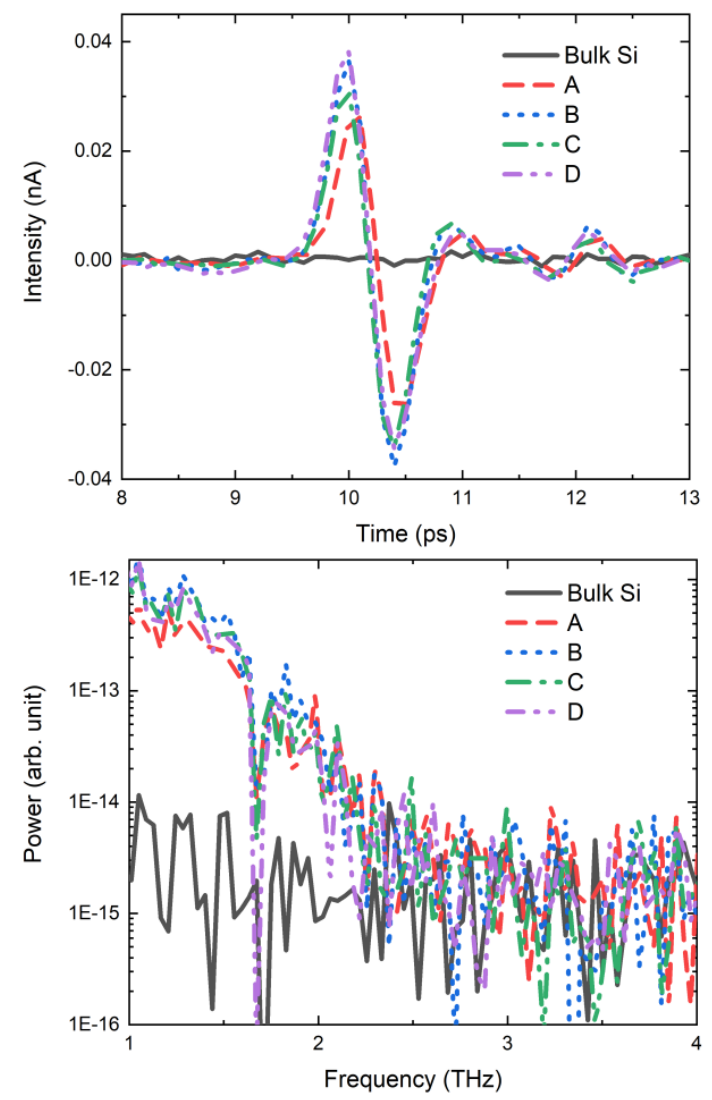

Fig. 3: THz-TDS emission intensity and power spectra of all GaAs on textured p-Si(100) samples compared to the bulk substrate. 
Table 2: Peak-to-peak $\mathrm{THz}$ emission intensities of the GaAs on textured p-Si(100) samples.

\begin{tabular}{cc}
\hline SAMPLE & THz peak-to-peak intensity \\
\hline A & $5.35 \times 10^{-2}$ \\
B & $7.24 \times 10^{-2}$ \\
C & $6.54 \times 10^{-2}$ \\
D & $7.41 \times 10^{-2}$ \\
\hline
\end{tabular}

\section{Summary}

Terahertz emission from GaAs on textured p-Si(100) was demonstrated. Reflectivity measurements indicated that the substrate etched for 60 minutes had the highest absorption due to the surface roughness. The Raman spectra of all the samples confirmed the growth of GaAs on textured p-Si(100). The $\mathrm{THz}$ emission intensities were comparable among all samples. Nevertheless, the GaAs grown on p-Si(100) textured for 60 minutes exhibited the most intense $\mathrm{THz}$ emission attributed to the increased absorption due to surface texturing. All samples had frequency bandwidths of $\sim 2.5 \mathrm{THz}$.

\section{Acknowledgements}

This work was sponsored in part by grants from UP Systems Grant, DOST-GIA, DOST-PCIEERD and CHED-PCARI (IIID-2015-13).

\section{References}

1. M. Tonouchi, "Cutting-edge terahertz technology," Nat. Photonics, vol. 1, no. 2, pp. 97-105, 2007.

2. S. S. Dhillon et al., "The 2017 terahertz science and technology roadmap," J. Phys. D. Appl. Phys., vol. 50, no. 4, 2017.

3. N. M. Burford, et al., "Review of terahertz photoconductive antenna technology," vol. 56, no. 1, 2017.

4. K. Sakai et al., "Terahertz Optoelectronics," in Topics in Applied Physics, vol. 97, Berlin, Heidelberg: Springer-Verlag, 2005.

5. R. F. C. Farrow, Molecular Beam Epitaxy: Applications to Key Materials. Park Ridge, New Jersey, USA: Noyes Publication, 1995.

6. Y. Kamo, et al., "Highly efficient photoconductive antennas using optimum low-temperature-grown GaAs layers and Si substrates Highly efficient photoconductive antennas using optimum lowtemperature-grown GaAs layers and $\mathrm{Si}$ substrates," Jpn. J. Appl. Phys., vol. 53, no. 3, p. 032201, 2014.

7. S. F. Fang et al., "Gallium arsenide and other compound semiconductors on silicon," J. Appl. Phys., vol. 68, no. 7, 1990.

8. Y. B. Bolkhovityanov and O. P. Pchelyakov, "GaAs epitaxy on Si substrates: modern status of research and engineering," Uspekhi Fiz. Nauk, vol. 178, no. 5, p. 459, 2008.

9. H. Morkoç et al., "High-Quality GaAs MESFET's Grown on Silicon Substrates by Molecular-Beam Epitaxy," IEEE Electron Device Lett., vol. 6, no. 7, pp. 381-383, 1985.

10. S. M. Koch, S. J. Rosner, R. Hull, G. W. Yoffe, and J. S. Harris, "The growth of GaAs on Si by MBE," J.
Cryst. Growth, vol. 81, no. 1-4, pp. 205-213, 1987.

11. M. Yamaguchi, M. Tachikawa, Y. Itoh, M. Sugo, and S. Kondo, "Thermal annealing effects of defect reduction in GaAs on Si substrates," J. Appl. Phys., vol. 68, no. 9, pp. 4518-4522, 1990.

12. N. Chand et al, "GaAs-on-Si: Improved growth conditions, properties of undoped GaAs, high mobility, and fabrication of high-performance AlGaAs/GaAs selectively doped heterostructure transistors and ring oscillators," J. Appl. Phys., vol. 67, no. 5, pp. 2343-2353, 1990.

13. P. Würfel. "Physics of Solar cells: from Principles to new Concepts". John Wiley \& Sons, 2008.

14. De Wolf. "Micro-Raman spectroscopy to study local mechanical stress in silicon integrated circuits." Semiconductor Science and Technology 11, vol. 139, no. 2, 1996.

15. G. Abstreiter, E. Bauser, A. Fischer, and K. Ploog, "Raman spectroscopy-A versatile tool for characterization of thin films and heterostructures of GaAs and AlxGa1-xAs," Appl. Phys., vol. 16, no. 4, pp. 345-352, 1978. 